\title{
Intraepithelial lymphocyte mitosis in a jejunal biopsy correlates with intraepithelial lymphocyte count, irrespective of diagnosis
}

\author{
ANNE FERGUSON AND K ZIEGLER \\ From the Gastrointestinal Unit, University of Edinburgh and Western General Hospital, Edinburgh
}

SUMMARY When there is villus atrophy in a jejunal biopsy, intraepithelial lymphocyte (IEL) mitosis correlates with a diagnosis of coeliac disease. We have examined the significance of IEL mitosis in jejunal biopsies with normal villi. Counts of IEL per 100 villus enterocytes, and IEL mitosis per $1000 \mathrm{IEL}$, were carried out in 81 jejunal biopsies. Thirty one were from patients with coeliac disease or dermatitis herpetiformis, and many of these, from treated patients, were histologically normal; 40 were from patients with other diagnoses, selected to include biopsies with a high IEL count (>40 IEL per 100 enterocytes) but normal villi. Three coeliacs and 10 dermatitis herpetiformis patients had an IEL count of less than 40 , and no IEL mitoses were found in these biopsies. Two dermatitis herpetiformis patients had IEL counts of $43.7 \%$ and $43.9 \%$, with no IEL mitoses, but in all other coeliac and dermatitis herpetiformis biopsies high IEL counts were associated with IEL mitotic indices between $0.05 \%$ and $1.77 \%$. In the noncoeliac, non-dermatitis herpetiformis group, no IEL mitoses were found in the 22 biopsies with IEL count less than $43 \%$. In the others, IEL counts ranged from $44 \cdot 8 \%$ to $127 \cdot 0 \%$, and IEL mitoses were present, with mitotic indices ranging from $0 \cdot 06 \%$ to $0 \cdot 49 \%$. This work shows that IEL mitosis in a jejunal biopsy is not specific for coeliac disease, but occurs whenever there is an increased density of IEL within the villus epithelium.

In untreated coeliac disease, intraepithelial lymphocytes (IEL), fewer in number than normal, are packed into the flat surface epithelium so that the count of lymphocytes per 100 villus or luminal enterocytes is high. ${ }^{1}$ These coeliac IEL are larger than IEL of non-coeliacs, many are crossing the basement membrane, and some are in mitosis. ${ }^{2}$ Mitotic properties of IEL have been examined in detail by Marsh, who suggests that a high mitotic index of IEL $(>0.2 \%)$ is a pathological feature specific for gluten sensitive enteropathies. He found that IEL mitotic index clearly distinguished coeliac disease from other conditions with flattening of the jejunal mucosa and malabsorption, ${ }^{3}$ and that a low IEL mitotic index identified patients with a flat jejunal mucosa which was not due to coeliac disease. $^{4}$

A high count of IEL is occasionally found in an otherwise normal jejunal biopsy - that is, where there are normal villi, crypts, epithelium and lamina

Address for correspondence: Dr Anne Ferguson, Gastro-Intestinal Unit, Western General Hospital, Crewe Road, Edinburgh EH4 2XU.

Received for publication 28 August 1985. propria infiltrate. ${ }^{5}$ In our experience (unpublished), this is not uncommon in gluten free diet treated coeliac patients, and a high IEL count is also found from time to time in non-coeliac patients. We have tested Marsh's hypothesis, that IEL mitosis is specific to coeliac disease, by comparing biopsies from patients with coeliac disease or dermatitis herpetiformis, with those from other patients in whom, despite normal villi, a high IEL count had been noted. We have also examined the correlation between IEL count and mitotic index of IEL.

\section{Methods}

SELECTION OF BIOPSIES FOR STUDY

Some 250-400 jejunal biopsies are carried out each year in this Gastrointestinal Unit. Specimens are fixed in formal-saline, paraffin embedded, and in addition to the diagnostic histopathological assessment by a pathologist, an H\&E stained section of each biopsy is screened by one of us (AF). A final diagnosis is recorded later, after all investigations have been completed. Various categories required 
for this project were defined (see below) and biopsies selected for further study if the specimen was well orientated and there was a substantial amount of tissue remaining in the block.

The following groups of specimens were selected: (a) Biopsies from treated coeliacs, with normal villus and crypt architecture and normal columnar villus enterocytes (10 biopsies) or with partial villus atrophy (six biopsies). All the patients had had a typical jejunal biopsy at the time of diagnosis, and improvement in biopsy histology after three to six months treatment with a gluten free diet. Biopsies for this project had been taken when the patient was on a gluten free diet. (b) An unselected series of biopsies from patients with dermatitis herpetiformis, who were undergoing a full dietary, nutritional, and biopsy reassessment (Gawk-Rodger, Barnetson, and Ferguson, in preparation). (c) Biopsies previously indentified as having a high IEL count, with villi and crypts recorded as normal by a consultant pathologist and independently by one of us. A firm diagnosis was required, with the confident elimination of coeliac disease from the differential diagnosis. A similar number of biopsies, considered to be normal in all respects, was also selected. In all there were 40 non-coeliac, non-dermatitis herpetiformis biopsies, from patients with the following diagnoses: giardiasis - five (confirmed by presence of Giardia in jejunal mucus), traveller's diarrhoea - four, irritable bowel syndrome - four, normal volunteers - six (members of staff without gastro-intestinal symptoms, who had a jejunal biopsy carried out as part of another research project). There were 21 patients with other conditions including atopic eczema, ulcerative colitis, Crohn's disease, nutritional folate deficiency, lymphoma, systemic lupus erythematosus, aphthous ulceration, oesophagitis.

\section{TISSUE PROCESSING}

The paraffin embedded blocks were recut in serial sections $3 \mu \mathrm{m}$ thick, and stained with haematoxylin and eosin.

COUNTS OF IEL AND OF IEL MITOSES

Sections were examined with a Leitz microscope using $\times 12.5$ eyepieces and $\times 100$ oil objective. The count of IEL per 100 enterocytes was established by a differential count of cells of the villus epithelium. At least 1000 villus enterocytes were counted. For IEL mitoses, counts were done on every eighth section in the strip. When possible, 2000 or more IEL were examined, and the number of mitotic figures recorded. Counts were confined to the villus epithelium, and areas near crypt mouths were avoided.

\section{Results}

Biopsies from 81 patients were examined. In 68 , villi and crypts appeared normal. Biopsies from six of the coeliacs and seven dermatitis herpetiformis patients were distinctly abnormal with short villi, long crypts, characteristic lamina propria cellular infiltrate and high IEL count.

Intraepithelial lymphocyte counts were readily performed in all of the biopsies. In 72, there was sufficient material to allow counts of mitosis in 2000 IEL. In nine, which were relatively small, the mitotic index was based on the examination of between 1100 and 1800 IEL. In all of these the count of IEL mitoses was $0.0 \%$, and all had a normal IEL count.

JEJUNAL BIOPSIES FROM TREATED COELIACS

The results of measurements in the 16 biopsies from coeliac patients are given in Figure 1. The six biopsies with partial villus atrophy all had mitotic indices of 0.2 per 100 IEL or higher, and IEL counts ranged from 42 to 91 IEL per 100 enterocytes. In the 10 biopsies with normal villi, IEL counts varied from 28 to 79 . None of the three biopsies with a normal IEL count $(<40)$ had any IEL in mitosis. In all of the others, IEL mitotic indices were $0.05 \%$ or higher. There was a significant correlation between IEL count and IEL mitotic index both for the group of abnormal biopsies $(r=0 \cdot 87)$ and for those with normal villi $(r=0 \cdot 84)$. Although the mitotic index of the abnormal biopsies tended to be higher, for a given IEL count, than that of the biopsies with normal villi, there was no significant difference between the regression lines for the two groups.

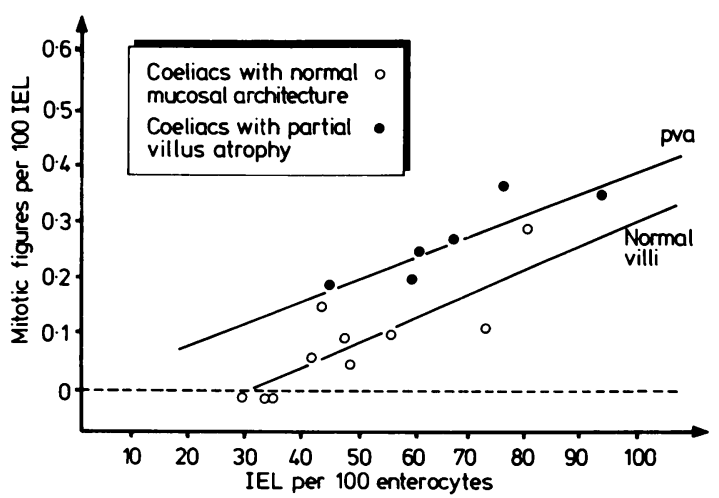

Fig. 1 Counts of IEL, and of IEL in mitosis, in jejunal biopsies from 16 patients with coeliac disease. All were taking a gluten free diet. Regression lines are shown separately for the group of biopsies with normal villi and those with partial villus atrophy. 


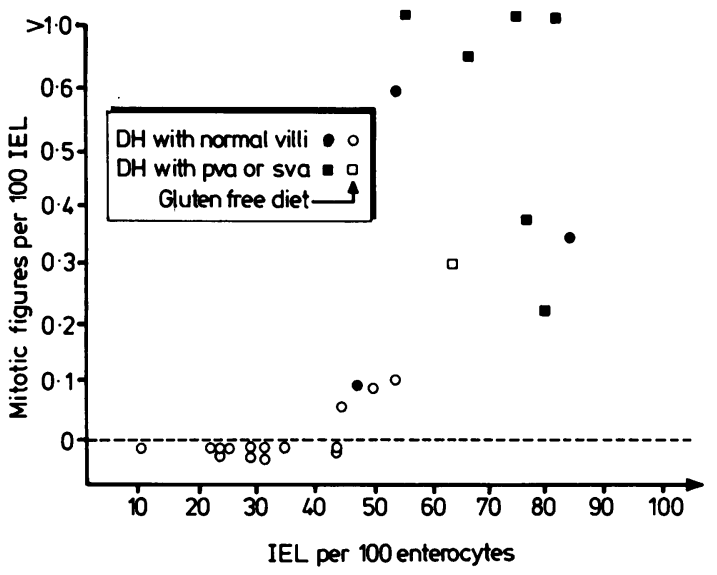

Fig. 2 Counts of IEL and of IEL in mitosis, in jejunal biopsies from 25 patients with dermatitis herpetiformis. Open circles and squares indicate patients taking a gluten free diet, and biopsies with normal villi and with abnormal architecture are separately identified.

\section{JEJUNAL BIOPSIES FROM DERMATITIS}

HERPETIFORMIS PATIENTS

Fifteen of the dermatitis herpetiformis patients were on a strict gluten free diet. Ten were eating gluten (8-20 g daily), of whom six had partial or subtotal villus atrophy, as did one of the gluten-free diet patients. Results for these biopsies are given in Figure 2. Irrespective of villus atrophy or diet status, there is a clearcut relationship between IEL count and mitotic index, and there were no mitoses in the 12 biopsies with IEL count of less than $44 \%$.

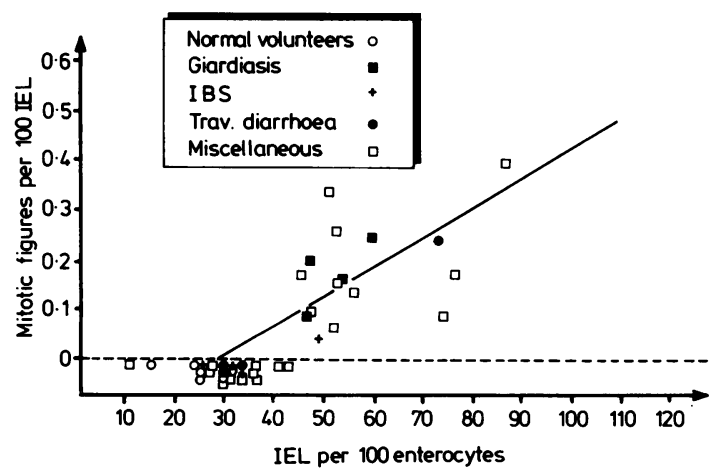

Fig. 3 Counts of IEL and of IEL in mitosis, in jejunal biopsies from 40 subjects or patients with diagnoses as shown.
JEJUNAL BIOPSIES FROM PATIENTS WITH OTHER DIAGNOSES

Results are shown in Figure 3. All six normal volunteers had IEL counts well within the accepted normal range and in none was any mitotic figure found within the IEL population. Indeed of the 23 biopsies with IEL count less than 43 , no mitotic figure of an IEL was found. On the other hand, irrespective of the diagnosis, all of the 17 biopsies from patients with IEL counts greater than 43 had an IEL mitotic index $0.05 \%$ or higher. The correlation co-efficient of IEL mitosis against IEL count was 0.84 ; the slope of the regression line was significantly different from that of both groups of coeliac biopsies.

\section{Discussion}

The function of lymphocytes within the small bowel epithelium is unknown, but examination of their numbers and properties is valuable in the investigation of patients with intestinal disease. The density of intraepithelial lymphocyte infiltrate in the epithelial microenvironment can be measured by a differential count of epithelial and intraepithelial cells, ${ }^{5}$ whereas the total number of IEL in a segment of the intestine is established by counting all of the cells in epithelium projected from a unit length or area of muscularis mucosae. ${ }^{26}$ An IEL count can be done in about 10 minutes, whereas two hours, and semi-thin sections, are required for a count of IEL mitoses. It is, therefore, important to establish which clinical situations require the measurement of IEL mitotic index. We have found a close relationship between IEL count and IEL mitotic index, and so there is little to be gained from counts of IEL mitoses in routine diagnostic work. In the present state of knowledge, however, we recommended that both investigations be done as part of the detailed analysis of the jejunal mucosa in those patients with villus atrophy in whom the diagnosis of coeliac disease is considered to be unlikely. More work is needed to establish whether counts of IEL mitoses are useful in the diagnosis of other food protein sensitive enteropathies, including those in domestic animals.

It is feasible, although time consuming, to count IEL mitoses in routinely obtained, formalin fixed, paraffin embedded, jejunal biopsies. We found that the marginal improvement in magnification obtained by using $\times 12.5$ instead of $\times 10$ eyepieces allowed satisfactory assessment of mitoses with a standard Leitz microscope. Large, well orientated specimens were selected, so that long strips of $3 \mu \mathrm{m}$ serial sections could be recut. Despite this, in nine of the 81 biopsies there was insufficient material to allow examination of 2000 IEL. 
Most workers report normal values for IEL count in the range 10-40 IEL per 100 villus enterocytes. ${ }^{1}$ We did not set out to establish a normal range for IEL mitotic index, and most of the biopsies used were from patients with gastrointestinal symptoms or diseases. The complete absence of IEL mitoses in jejunal biopsies from six healthy adult volunteers, however, tends to suggest that IEL in mitosis are a pathological feature of the jejunum, even if the mitotic index is fairly low. Indeed the majority of non-coeliac subjects studied by Marsh had an IEL mitotic index of $0.0 \% .^{34}$

The limited study we have carried out in biopsies from coeliac and dermatitis herpetiformis patients confirms that both IEL mitotic index and IEL count are high in treated patients who still have partial villus atrophy. Although there were a number of gluten free diet treated patients with normal villi, normal IEL count and no IEL mitoses, some had high IEL counts, of similar magnitude to the patients with partial villus atrophy. On the other hand, there was a tendency for the IEL mitotic index to be substantially higher in the dermatitis herpetiformis patients on a gluten containing diet than in others, and the index was also higher in coeliac biopsies with partial villus atrophy when compared with the group of biopsies with normal villi. The shifts in relationship between IEL count and IEL mitotic index with diet and with improvement in villus architecture, deserve further investigation.

The results of greatest interest are those obtained in the heterogeneous group of non-coeliac patients with high IEL counts but otherwise normal biopsies. Clearly, mitosis of IEL is not a feature specific for coeliac disease but occurs in all of the conditions in which IEL are infiltrating the epithelium in large numbers. The cut off point at an IEL count of $43 \%$ suggests that values above this are indeed abnormal, and not merely at the top end of the normal range. Indeed, we have recently reported that intestinal permeability is abnormal in similar patients with a high IEL count but normal villi. ${ }^{7}$ It is not known whether the lymphocyte infiltrate causes the abnormal permeability or whether the two are unrelated but parallel manifestations of a subtle abnormality in intestinal anatomy and function.

An increase in the IEL count is a consistent feature of cell mediated immune reactions in the small intestine of experimental animals. This occurs within 24 hours of induction of a graft-versus-host reaction $(\mathrm{GvHR})$ in neonatal mice, ${ }^{8}$ and changes in IEL count may be the only manifestation of intestinal sensitisation to the protein antigen ovalbumin. ${ }^{9}$ Studies on the pathogenesis of the intestinal lesion in GvHR tend to suggest that events occurr- ing in the lamina propria, involving $\mathrm{T}$ helper cells and lymphokine secretion, precede and cause the rise in IEL count.$^{10}$ Nevertheless, mitotic activity of IEL, as measured by a metaphase accumulation technique, is increased in mice with GvHR. ${ }^{11}$

If high IEL counts and IEL mitosis are indeed markers of a mucosal delayed type hypersensitivity reaction, the results described above further support a role for $T$ cells in the pathogenesis of the enteropathy in coeliac disease and giardiasis. Less severe intestinal damage in patients with diarrhoea, eczema etc. may also be $T$ cell mediated, but the antigens concerned are unknown. Candidates will include autoantigens, viruses, commensal bacteria, small protozoa, and foods other than gluten.

We would like to thank the nursing staff of the Clinical Investigation Suite in the GI Unit, who carried out jejunal biopsies, and Mr A Sutherland for technical assistance with tissue processing. We are also grateful to the medical and scientific staff of the Department of Pathology at the Western General Hospital, for their continuing collaboration and interest in our research. This work has been supported by a grant from the Deutsche Forschungsgemeinschaft.

\section{References}

1 Ferguson A. Intraepithelial lymphocytes of the small intestine. Gut 1977; 18: 921-37.

2 Marsh MN. Studies of intestinal lymphoid tissue. III. Quantitative analyses of epithelial lymphocytes in the small intestine of human control subjects and of patients with celiac sprue. Gastroenterology 1980; 79: 481-92.

3 Marsh MN. Studies of intestinal lymphoid tissue. IV. The predictive value of raised mitotic indices among jejunal epithelial lymphocytes in the diagnosis of gluten-sensitive enteropathy. J Clin Pathol 1982; 35: 517-25.

4 Marsh MN, Haeney MR. Studies of intestinal lymphoid tissue. VI. Proliferative response of small intestinal epithelial lymphocytes distinguishes gluten- from nongluten-induced enteropathy. J Clin Pathol 1983; 36: 149-60.

5 Ferguson A, Murray D. Quantitation of intraepithelial lymphocytes in human jejunum. Gut 1971; 12: 988-94.

6 Corazza GR, Frazzoni M, Gasbarrini G. Jejunal intraepithelial lymphocytes in coeliac disease: are they increased or decreased? Gut 1984; 25: 158-62.

7 Strobel S, Brydon WG, Ferguson A. The cellobiose/ mannitol sugar permeability test complements biopsy histopathology in clinical investigation of the jejunum. Gut 1984; 25: 1241-6.

8 Mowat AMcI, Ferguson A. Intraepithelial lymphocyte 
count and crypt hyperplasia measure the mucosal component of the graft-versus-host reaction in mouse small intestine. Gastroenterology 1982; 83: 417-23.

9 Strobel S, Ferguson A. Modulation of intestinal and systemic immune responses to a fed protein antigen, in mice. Gut In press.
10 Mowat AMcI, Ferguson A. Hypersensitivity reaction in the small intestine. 6. Pathogenesis of the graftversus-host reaction in the small intestinal mucosa. Transplantation 1981; 32: 238-43.

11 Ferguson A, Ziegler K, Strobel S. Gluten intolerance (coeliac disease). Ann Allergy 1984; 53: 637-42. 